\title{
Crack-Syndrom: Pulmonale Komplikationen nach Kokaininhalation
}

Kasuistik und Darstellung des Krankheitsbildes

\author{
T. O. Hirche ${ }^{1}$ \\ E. Lambrecht ${ }^{2}$ \\ T. O. F. Wagner ${ }^{1}$
}

\author{
Crack-Syndrome: The Pulmonary Complications of Inhaled Cocaine. \\ A Review a Propos a Case Report
}

\section{Zusammenfassung}

Wir berichten über einen 45-jährigen Patienten mit Husten, Hämoptysen, thorakalen Schmerzen und neu aufgetretenen pulmonalen Infiltraten beidseits. Die bronchoalveoläre Lavage und transbronchiale Biopsien ergaben die Infiltration mit neutrophilen Granulozyten sowie die Ausbildung von Fremdkörpergranulomen. Ursächlich wurde ein Crack-Syndrom infolge eines inhalativen Kokainabusus diagnostiziert und der Patient zeigte eine rasche Besserung unter symptomatischer Therapie und temporärer Noxenkarenz. Als Crack wird ein hitzestabiles Derivat des Kokains bezeichnet, welches tabakähnlich inhaliert werden kann, wobei die Lunge das primär exponierte und geschädigte Organ darstellt. Neben den geschilderten Symptomen wurden ausgeprägte ischämisch/toxische Schädigungen der Alveolarwände und des pulmonalen Gefäßbettes beschrieben. In der Folge kann sich ein primäres Lungenödem bis hin zum Vollbild eines ARDS entwickeln. Da der Crack-Konsum in Deutschland eine kontinuierliche Zunahme aufweist und Crack in einzelnen Regionen bereits zu den am häufigsten verwendeten illegalen Substanzen zählt, ist in Zukunft gehäuft mit dem Auftreten des beschriebenen Syndroms zu rechnen.

\section{Abstract}

A 45-year-old patient developed shortness of breath, intensive cough, hemoptysis, chest pain and acute bilateral pulmonary infiltrates following the inhalation of crack-cocaine. The bronchoalveolar lavage and transbronchial biopsy revealed infiltrations of polymorphnuclear neutrophils and the formation of foreign body granulomas. The diagnosis of a crack-syndrome was made and the patient rapidly improved under temporary discontinuation of cocaine inhalation and symptomatic therapy. Crack-cocaine is the free-base of cocaine-hydrochloride and its chemical properties allows it to be inhaled tobacco-like. Therefore the lungs become the principal organs exposed and affected. In addition to our findings, diffuse damage of the alveolar wall and capillary injury due to vasoconstriction and toxic action were reported, in some cases rapidly progressing into pulmonary oedema and ARDS. As the consumption of crack cocaine in Germany has markedly increased over the past decade, a higher prevalence of the reported syndrome has to be assumed.

\section{Fallbericht}

\section{Anamnese}

Ein 45-jähriger Patient wurde wegen seit 4-6 Wochen bestehendem, als quälend empfundenem Reizhusten auf unserer pneumologischen Station vorgestellt. Gelegentlich wurde Blut im Sputum beobachtet. Weiterhin beklagt der Patient eine zunehmende Dyspnoe, zuletzt bereits bei leichter Belastung, sowie atemabhängige thorakale Schmerzen. Wiederholt seien Temperaturerhöhungen und Nachtschweiß aufgetreten. Anamnestisch

Institutsangaben

${ }^{1}$ Zentrum für Innere Medizin II, Schwerpunkt Pneumologie und Allergologie

(Leiter: Prof. Dr. med. T. O. F. Wagner)

2Zentrum für Pathologie (Direktor: Prof. Dr. med. M.-L. Hansmann)

Klinikum der Johann-Wolfgang-Goethe-Universität Frankfurt

Korrespondenzadresse

Dr. med. T. O. Hirche · Schwerpunkt für Pneumologie und Allergologie • Medizinische Klinik II, Haus 11 .

Johann-Wolfgang-Goethe-Universität Frankfurt · Theodor-Stern-Kai 7 · 60590 Frankfurt am Main

E-mail: t.hirche@em.uni-frankfurt.de

Bibliografie

Pneumologie 2002; 56: 684-688 @ Georg Thieme Verlag Stuttgart · New York · ISSN 0934-8387 
fanden sich eine 1989 erstdiagnostizierte HIV-Infektion bei einem bis 1991 bestehenden intravenösen Drogenabusus sowie eine chronisch persistierende Hepatitis-C-Virusinfektion. Der Patient stellte sich seit einigen Jahren in unregelmäßigen Abständen in unserer HIV-Ambulanz vor. Eine AIDS-definierende Erkrankung wurde bisher nicht diagnostiziert; im Monat vor der stationären Aufnahme wurde die HI-Viruslast mit 73000 Kopien/ml bei einer Helferzellzahl von $200 / \mu l$ bestimmt.

Eine antiretrovirale Therapie wurde mehrfach begonnen, jedoch wegen subjektiv schlechter Verträglichkeit wieder abgesetzt. Die Einnahme von Trimethoprim/Sulfamethoxazol (160/800 mg oral, $3 \times$ /Woche) zur Prophylaxe einer Pneumocyctis-carinii-Pneumonie (PCP) wurde nur unregelmäßig durchgeführt. Seit 1991 erfolgte eine Substitutionstherapie mit derzeit $185 \mathrm{mg} / \mathrm{d}$ Methadon per os, ein weiterer Beikonsum wurde verneint. Der Patient wirkte sozial gut rehabilitiert und war zuletzt als gelernter Raumausstatter beschäftigt. Alkohol wurde in Form mehrerer Flaschen Bier/Tag konsumiert, es bestand ein fortgesetzter Nikotinabusus von etwa 20 packyears.

\section{Klinischer Untersuchungsbefund}

Leicht reduzierter Allgemeinzustand bei adipösem Ernährungszustand. Unauffälliger Herz- und Gefäßstatus, kein Hinweis auf fortgesetzte i.v.-Injektionen. Vesikuläres Atemgeräusch, bds. basal betont feinblasige Rasselgeräusche, Klopfschall sonor, Lungengrenzen bds. $2 \mathrm{~cm}$ atemverschieblich. Die übrige internistische/neurologische Untersuchung ergab unauffällige Befunde.

\section{Laborbefunde}

BSG $39 \mathrm{~mm} / \mathrm{h}(<20)$, CRP 2,2 mg/dl $(<0,5), \mathrm{Hb} 13,6 \mathrm{~g} / \mathrm{dl}(14-18)$, Leukos 4,4/nl (4,8 - 11,0) GOT $23 \mathrm{U} / \mathrm{l}(<18)$, GPT $23 \mathrm{U} / \mathrm{l}(<28)$, LDH 254 U/l (<240). Hepatitisserologie: HBV-Antikörper (anti-HBC) pos., HBs-Antigen neg., HCV-Antikörper pos. Die übrigen serumchemischen Parameter lagen im Normbereich. Der Tuberkulinhauttest nach Mendel-Mantoux war bis zur Stufe GT-10 negativ.

\section{Apparative Diagnostik}

Diskrete Hepatomegalie, ansonsten unauffällige thorako-abdominale Sonographie. Lungenfunktionell zeigte sich eine leichte, peripher betonte Obstruktion (Vitalkapazität 4,86 [89\% des Solls], $\mathrm{FEV}_{1}: 3,7$ Liter [ $88 \%$ des Solls], Tiffeneau-Test $76 \%$ ) bei leicht erhöhten Atemwegswiderständen (SR tot 1,46 kPa*s [124\% des Solls]) ohne Hinweis auf eine pulmonale Überblähung oder restriktive Ventilationsstörung (TLC 7,82 Liter [101,5\% des Solls]). Blutgasanalysen in Ruhe ergaben eine leichte respiratorische Partialinsuffizienz $\left(\mathrm{p}_{\mathrm{a}} \mathrm{O}_{2} 63-73 \mathrm{~mm} \mathrm{Hg}\right)$, welche unter Belastung diskret verstärkt wurde.

Im Röntgen-Thorax fand sich beidseits eine disseminierte, feinfleckige Zeichnungsvermehrung. Im daraufhin durchgeführten HR-CT wurde, bei grenzwertig vergrößerten mediastinalen Lymphknoten, eine milchglasartige Verschattung der sekundären Lobuli, vereinbar mit einer Alveolitis, beschrieben (Abb.1).

\section{Arbeitsdiagnose}

In der Zusammenschau der Befunde, insbesondere unter Berücksichtigung der radiologischen Beurteilung, stellten wir die Verdachtsdiagnose einer subakut verlaufenden Pneumocyctis-carinii-Pneumonie (PCP). Differenzialdiagnostisch zogen wir, trotz
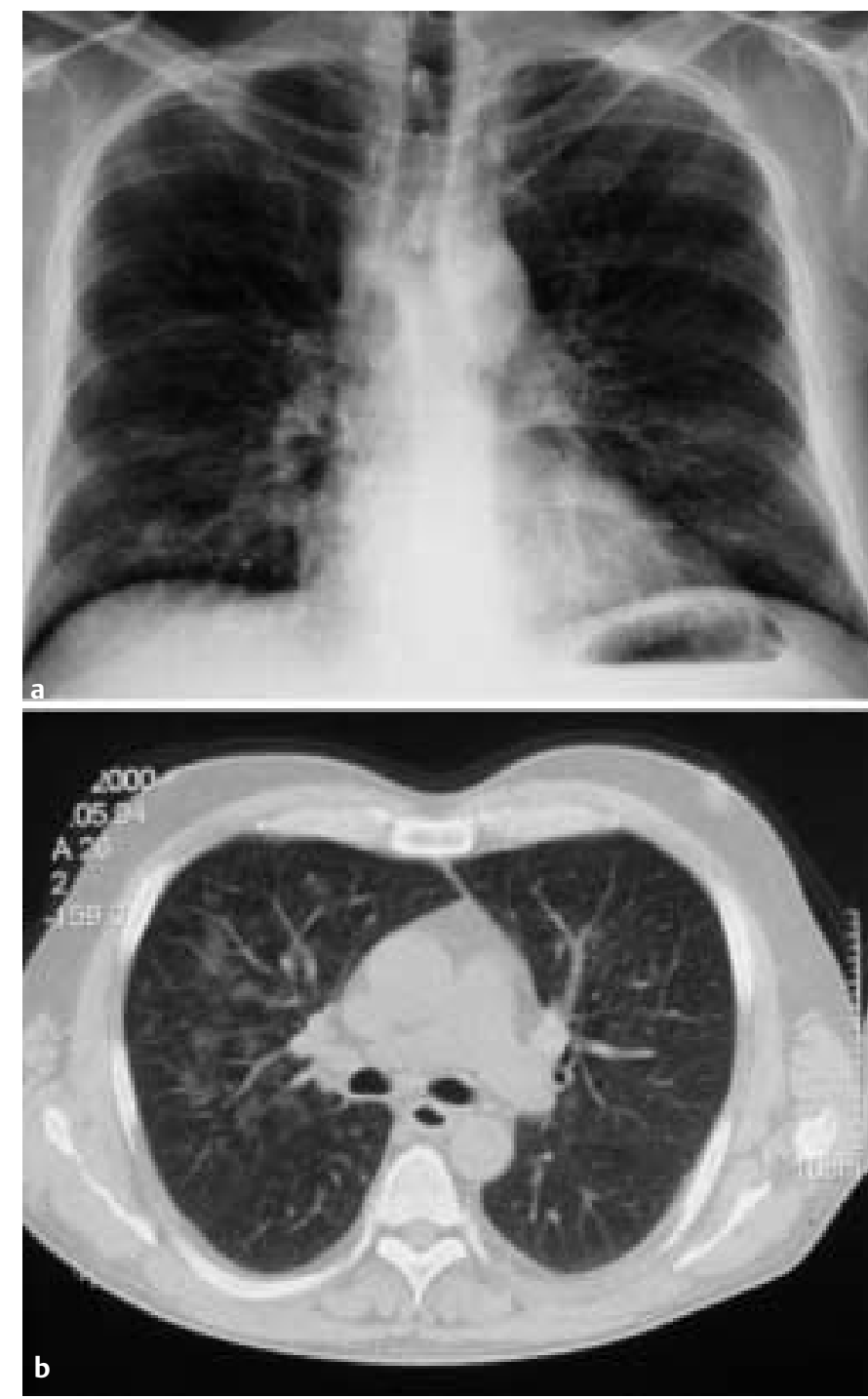

Abb. 1 Radiologische Befunde: a Der Röntgen-Thorax weist beidseits eine disseminierte, basal betonte, feinfleckige Zeichnungsvermehrung auf. b Im HR-CT zeigt sich ubiquitär (hier rechtsbetont) das Bild einer Alveolitis mit milchglasartiger Verschattung der sekundären Lobuli. Grenzwertig vergrößerte mediastinale Lymphknoten.

Anergie im Tuberkulintest, bei Nachtschweiß und vergrößerten mediastinalen Lymphknoten eine Tuberkulose in Betracht.

\section{Ergänzende Untersuchungen}

Eine zur Diagnosesicherung durchgeführte Bronchoskopie ergab neben diskreten Zeichen einer Bronchitis und leicht vermehrtem weißlichen Sekret einen unauffälligen endobronchialen Befund. Die bronchoalveoläre Lavage (BAL) wies eine leichte Erhöhung des Neutrophilenanteils (17,5\%), sowie eine Mischflora ohne Nachweis spezifischer Erreger auf, insbesondere fanden sich weder Pneumozysten noch säurefeste Stäbchen. Die transbronchiale Biopsie des rechten Oberlappens zeigte alveoläres Parenchym mit fokaler intraalveolärer Makrophagenansammlung, Siderophagen und mehrkernigen Fremdkörperriesenzellen (Abb.2). Da durch den Pathologen differenzialdiagnostisch auf eine inhalative Noxe verwiesen wurde, initiierten wir ein Drogenscreening im Urin des Patienten und wiesen neben Cannabis (62 ng/ml) auch Kokain $(1000 \mathrm{ng} / \mathrm{ml})$ nach. Mit den Untersuchungsergeb- 


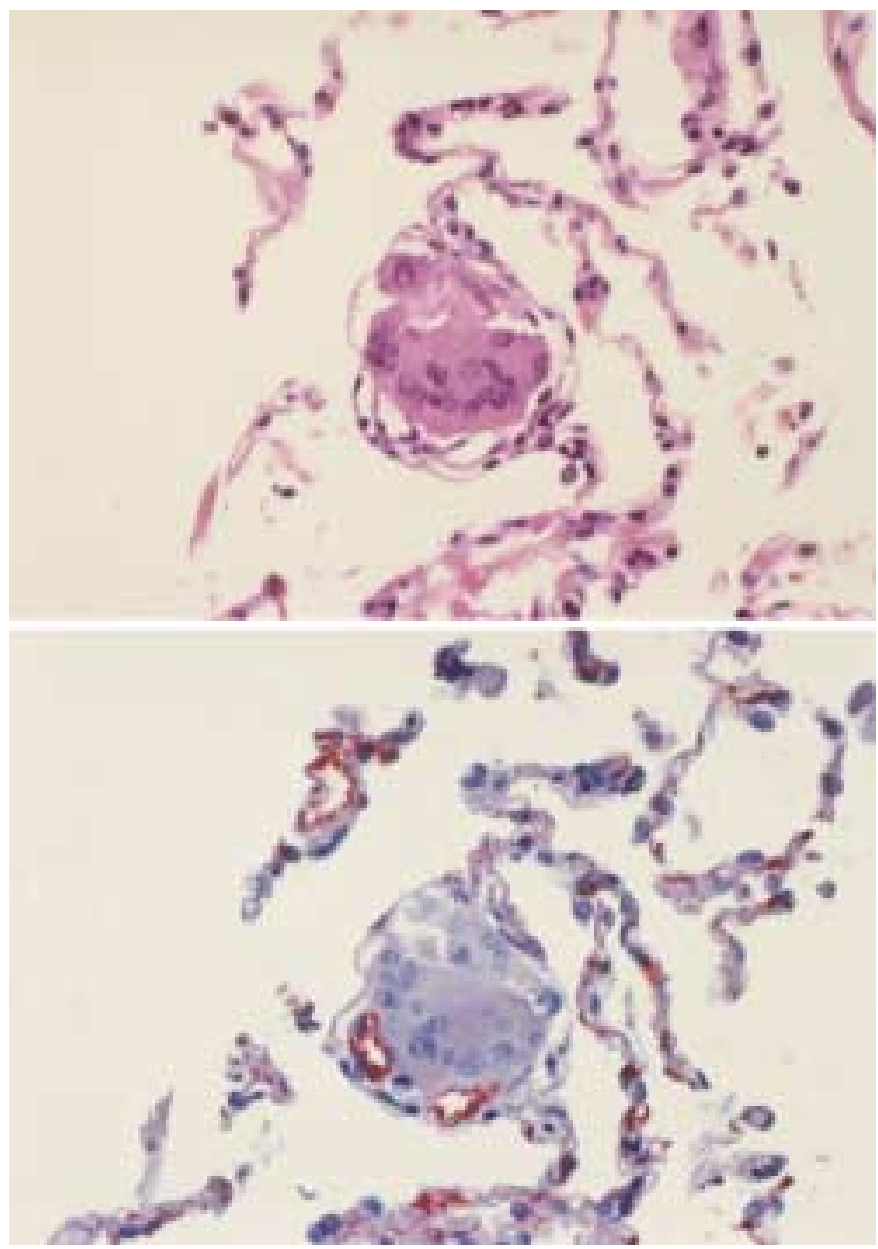

Abb. 2 Histologie aus der transbronchialen Lungenbiopsie: a Eine von zahlreichen mehrkernigen Riesenzellen vom Fremdkörpertyp mit kleinen Einschlüssen. Schmale, nicht entzündlich infiltrierte Alveolarsepten (HE 400×). b Die immunhistochemische Endotheldarstellung durch Antikörper gegen Faktor-VIII-assoziiertes Antigen (rot) zeigt die intraalveoläre, extravaskuläre Lokalisation der Riesenzellen als Hinweis auf eine inhalative Noxe $(400 \times)$.

nissen konfrontiert, gab der Patient an, seit einigen Monaten Kokain in Form von „Crack“ zu inhalieren.

\section{Diagnose}

Wir stellten die Diagnose eines „Crack-Syndroms“, in der englischsprachigen Literatur auch als „Crack-Lung“ bezeichnet. Es fasst eine Vielzahl vorwiegend pulmonaler Komplikationen zusammen, welche als Folge der Inhalation von Crack-Kokain auftreten können. Aufgrund der weltweit dramatisch zunehmenden Verbreitung dieser Rauschdroge, ist in Zukunft auch in Europa vermehrt mit dem beschriebenen Krankheitsbild zu rechnen [1-3].

Unter einer mehrtägigen Noxenkarenz und symptomatischer Therapie war eine deutliche klinische Verbesserung und ein Rückgang der infiltrativen Veränderungen zu beobachten. Die weitere Anbindung in ein stationäres Entzugsprogramm wurde abgelehnt, so dass wir den Patienten mit der dringenden Empfehlung entließen, sich zur Rückfallprophylaxe in weitere suchttherapeutische Betreuung zu begeben.

\section{Crack-Kokain: Verbreitung und Konsummuster}

Crack wurde Ende der 80er-Jahre aus Kokain entwickelt und gehört in den USA heute bereits zu den häufigst verwendeten illegalen Substanzen. Umfragen an US-Highschools ergaben bei 6\% der Befragten einen gelegentlichen Konsum von Kokain, 30\% davon verwendeten Kokain in Form von Crack [1,4].

Das aus der Pflanze Erythroxylon coca gewonnene Alkaloid Kokain liegt üblicherweise als Hydrochloridverbindung in Form eines feinkörnigen weißen Pulvers vor. Zur Verwendung als Rauschdroge wird es intravenös oder über die Nasenschleimhaut appliziert. Wegen einer fehlenden Hitzestabilität eignet sich das Kokain-Hydrochlorid nicht zur Inhalation über Rauch.

Rauchbar, weil hitzebeständig, ist jedoch die freie Base des Kokains (engl. free-base). Die eigene Herstellung durch die Konsumenten ist technisch einfach möglich, wobei die Hydrochloridbindung chemisch durch Zugabe von Natrium-Bicarbonat (Na$\mathrm{HCO}_{3}^{-}$), handelsüblich als Backpulver zu beziehen, gespalten wird. Die präzipitierende freie Base des Kokains wird mit Äther oder Ethanol zu einer elastischen Masse vermischt, nach Aushärtung durch Trocknung erhält man einen Kristall, welches als rock-cocain oder stone bezeichnet wird (Abb. 3). Die Substanz wird in speziellen Pfeifen inhaliert oder mit Tabak oder Marihuana vermischt und geraucht, das bei der Verbrennung entstehende knackende Geräusch (to crackle = knistern) führte zur Benennung der Droge als Crack [5]. Die freigesetzten Kokainpartikel messen durchschnittlich 2,3 $\mu \mathrm{m}$ und sind damit nach Inhalation gut alveolengängig [6]. Aufgrund der großen alveolaren Oberfläche erfolgt die unverzügliche Resorption mit der Folge des schnellen Anflutens der Substanz in der zerebralen Zirkulation. Der maximal euphorisierende Effekt nach Inhalation von CrackKokain tritt bereits nach $6-8$ Sekunden auf, während er nach intravenöser Injektion erst nach 16-20 Sekunden, bei der Applikation über die Nasenschleimhaut sogar erst nach 3-5 Minuten zu beobachten ist $[2,7]$. Das schnelle Anfluten des Crack-Kokains stellt dabei auch eine wesentliche Erklärung für das ungewöhnlich hohe Suchtpotenzial der Substanz dar [2]. Bereits nach wenigen Minuten geht das empfundene Hochgefühl in einen Zustand

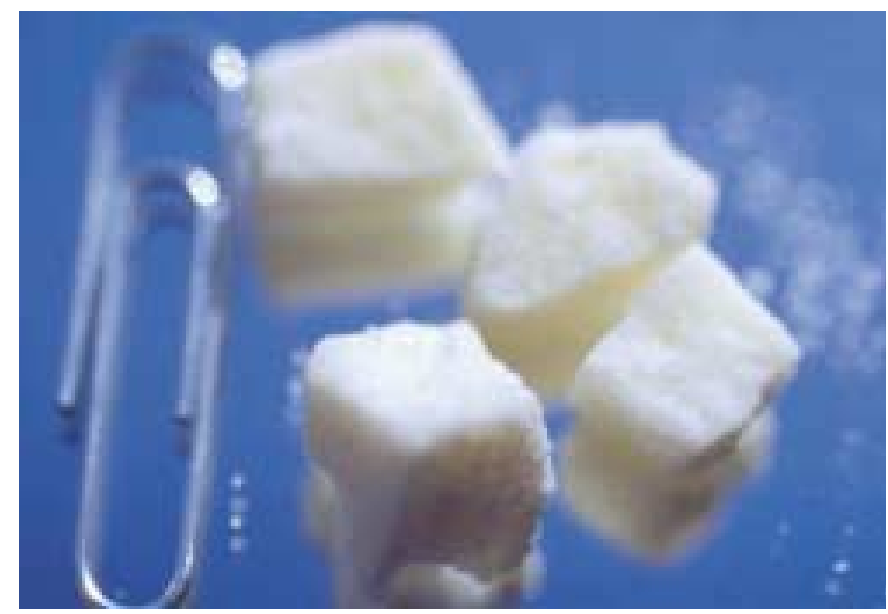

Abb. 3 Kristalle der freien Kokainbase, sog. Crack (mit freundlicher Genehmigung www.streetdrugs.org). 
ausgeprägter Dysphorie über (sog. Flash- und Crashphänomen) und verleitet zu einer schnellen Wiederbeschaffung der Droge, es wurden Konsumfrequenzen zwischen 4-19×/Tag beschrieben [3]. Durch den im Vergleich zum Kokain deutlich erniedrigten Marktpreis verschiebt sich die Verfügbarkeit in den Bereich sozial schwacher oder minderjähriger Konsumenten [8]. Eine im Auftrag der Bundesdrogenbeauftragten durchgeführte und im August 2001 veröffentlichte Bestandsaufnahme zum CrackKonsum in Deutschland zeigt eine kontinuierliche Zunahme mit einer regionalen Häufung in Frankfurt, Hamburg und Hannover [3]. In diesen Städten steht der Crack-Konsum nach dem Heroinkonsum bereits an zweiter Stelle der präferierten Drogen.

Dabei ist eine Zunahme des polyvalenten Drogengebrauchs zu beobachten, wobei bereits für ${ }^{2} / 3$ der Konsumenten harter Drogen ein zusätzlicher Crack-Abusus angenommen wird. In der polizeilichen Kriminalstatistik der Stadt Hamburg überstiegen die Delikte im Zusammenhang mit Kokain 1998 erstmals die in Verbindung mit Heroin, einhergehend mit einer deutlich gesteigerten Brutalität bei der Drogenbeschaffung. Besondere Schwierigkeiten werden von Einrichtungen der niedrigschwelligen Drogenhilfe im Umgang mit eingeschränkter Steuerungsfähigkeit, zunehmender Aggressivität und Verwahrlosung der Crack-Konsumenten berichtet $[3,9]$.

\section{Komplikationen nach Crack-Inhalation}

Während der Kokainabusus in der bisherigen Form über eine Stimulierung des Sympathikus-Tonus im Wesentlichen kardiovaskuläre Nebenwirkungen wie Tachykardie, Hypertonie, periphere Vasokonstriktion und Agitation bewirkt [10], stellt beim CrackAbusus die Lunge das primär exponierte und geschädigte Organ dar. Die Vielzahl der resultierenden pulmonalen Symptome wird als Crack-Syndrom oder Crack-Lunge beschrieben. Pulmonale Symptome nach Crack-Abusus (Tab.1) können innerhalb von Minuten bis zu einigen Stunden nach Inhalation auftreten [10]. Die häufigsten akuten respiratorischen Symptome stellen in bis zu 60\% der beschriebenen Fälle Husten mit Auswurf, Dyspnoe sowie thorakale Schmerzen dar [2]. Der Husten wird auf eine lokale Irritation der Atemwege nach Kontakt mit hohen Konzentrationen von Kokain zurückgeführt. Die gelegentlich beschriebene akut auftretende Obstruktion sowie Symptome einer exogen allergischen Alveolitis lassen sich am ehesten durch eine bronchiale Hyperreagibilität auf die im Inhalat enthaltenen Verunreinigungen (z.B. Backpulver) erklären [11]. Der thorakale Schmerz kann infolge des massiven Hustenreizes auftreten, differenzial-

Tab. 1 Pulmonale Komplikationen nach Crack-Inhalation

\footnotetext{
1. akute respiratorische Symptome

- Husten mit nicht purulentem Sputum

- Dyspnoe

- obstruktive Ventilationsstörung

- Thorakodynie

- Hämoptysen

2. physikalische Verletzungen

- Barotrauma/Pneumothorax

- thermische Schädigung/Verbrennung

3. Alveolitis/kryptogene organisierende Pneumonie

4. primäres Lungenödem/ARDS

5. pulmonale Hämorrhagie/Infarzierung
}

diagnostisch muss bei Kokainmissbrauch eine Myokardischämie bzw. ein Myokardinfarkt ausgeschlossen werden.

Aufgrund der speziellen Applikationsform des Crack-Kokains werden vermehrt physikalische Verletzungen im Bereich der Lunge beobachtet: Bei dem Versuch, durch tiefe Inhalation mit nachfolgendem Valsalva-Manöver, die Wirkung der Droge zu steigern, kann ein Barotrauma oder ein Pneumothorax entstehen. Durch Inhalation des heißen Rauches bzw. durch Verunreinigung mit brennbaren Lösungsmitteln (z.B. Äther) kann es zu gravierenden Verbrennungen im Bereich der Atemwege kommen [12].

Die in bis zu 30\% der Fälle beschriebenen Hämoptysen können durch Ruptur submuköser Atemwegsgefäße infolge mechanischer Schädigung (z. B. durch Hustenstöße) entstehen. Weiterhin bewirkt die durch Kokain induzierte Vasokonstriktion eine Schädigung des pulmonalen Gefäßbettes mit der Folge einer hypoxischen Wandschädigung. Auch ein direkt zytotoxischer Effekt von Kokain auf die Gefäßendothelien wird diskutiert [13].

Bei den Autopsien von Crack-Konsumenten, welche an den Folgen einer akuten Intoxikation verstorben waren, fanden sich neben intraalveolären Entzündungszellinfiltraten und Fremdkörpergranulomen Schädigungen der alveolo-kapillären Membranen mit akutem, nicht kardial bedingtem, interstitiellem Ödem und alveolären Hämorrhagien [14]. Das radiologische Korrelat bilden flüchtige, interstitielle und alveoläre Verschattungen. Diese stellen sich vorwiegend bilateral, perihilär und symmetrisch dar. Die Lungenfunktion erweist sich als wenig sensitiv und zeigt häufig einen unauffälligen Befund. Neben dem gehäuften Auftreten einer milden Obstruktion (bei regelmäßig bestehendem Nikotinabusus) findet sich vermehrt eine leichte Einschränkung der Diffusionskapazität [11].

Wenngleich sich die beschriebenen Symptome einschließlich der radiologischen Veränderungen in der Regel in 24 bis 72 Stunden nach Expositionsende zurückbilden, wird in seltenen Fällen eine hochgradige respiratorische Insuffizienz, gelegentlich verbunden mit der Notwendigkeit zur maschinellen Beatmung, beschrieben. Klinisch kann sich das Vollbild eines ARDS mit entsprechend hoher Mortalität entwickeln $[15,16]$. In Einzelfallberichten wird über pulmonale Infarzierungen sowie fulminante Hämorrhagien nach Crackinhalation berichtet $[14,17]$.

\section{Therapie}

Nach erfolgtem Abusus sollte ein engmaschiges Monitoring des Patienten über 24 Stunden durchgeführt werden, um die Entwicklung von Komplikationen (z. B. eines Lungenödems) frühzeitig zu erkennen. Milde respiratorische Symptome lassen sich häufig durch Gabe von Antiobstruktiva/Antitussiva bessern. Bei respiratorischer Partialinsuffizienz sollte die supportive Gabe von Sauerstoff erfolgen (Cave: Bei fortgesetztem Crack-Abusus wegen Entzündungsgefahr kontraindiziert). Über eine Gabe von Diuretika sowie Steroiden muss im Einzelfall entschieden werden, eine generelle Indikation oder Dosisempfehlung besteht nicht. Bei Entwicklung eines ARDS sollte entsprechend frühzeitig die Indikation zur Intubation und mechanischen Beatmung mit erhöhten PEEP-Werten gestellt werden. 
Die wichtigste Maßnahme besteht sicherlich in einer strikten Noxenkarenz. Eine besondere Schwierigkeit stellt dabei jedoch das ausgeprägte, vorwiegend psychisch bedingte Abhängigkeitspotenzial der Substanz dar. Zur Rückfallprävention sollte die Entlassung in eine psychosozial gestützte Weiterbetreuung (z. B. betreutes Wohnen, kognitiv orientierte Verhaltenstherapie, motivierende Gesprächstherapie) erfolgen. Stationäre Entgiftungsprogramme finden wenig Akzeptanz bei den Betroffenen und erscheinen in Kenntnis der dominierenden psychischen Abhängigkeit nicht indiziert. Präventive Maßnahmen (z.B. aufsuchende Sozialarbeit) wurden in Modellprojekten wie dem Crack-StreetProjekt in Frankfurt erprobt. Der kontinuierliche Zugang zu den Betroffenen wurde jedoch durch deren eingeschränkte Behandlungs- und Suchteinsicht sowie deren aufgehobenen Alltagsrhythmus erheblich erschwert. Substitutions- und Konsumeinrichtungen für Crack-Abhängige zur Reduktion von Suchtfolgeerkrankungen und Begleitkriminalität sind in den Niederlanden bereits in Erprobung, in Deutschland jedoch umstritten. In Frankfurt und Hamburg wurde dem sozialen Elend der Betroffenen durch die Einrichtung von Rückzugs- und Tagesruheräumen (sog. Chill-out-Zonen) begegnet $[3,9]$.

\section{Literatur}

${ }^{1}$ Benson MK, Bentley AM. Lung disease induced by drug addiction. Tho$\operatorname{rax} 1995 ; 50$ : $1125-1127$

${ }^{2}$ Haim DY, Lippmann ML, Goldberg SK et al. The pulmonary complications of crack cocaine. A comprehensive review. Chest 1995; 107: $233-240$

${ }^{3}$ Stoever H. Crack-Konsum in Deutschland: Verbreitung, Konsummuster, Risiken und Hilfsangebote. Bestandsaufnahme im Auftrag der Bundesdrogenbeauftragten. Universität Bremen, 2001

${ }^{4}$ Smart RG. Crack cocaine use: a review of prevalence and adverse effects. Am J Drug Alcohol Abuse 1991; 17: 13 - 26

${ }^{5}$ Khalsa ME, Tashkin DP, Perrochet B. Smoked cocaine: patterns of use and pulmonary consequences. J Psychoactive Drugs 1992; 24: $265-272$

${ }^{6}$ Snyder CA, Wood RW, Graefe JF et al. "Crack smoke" is a respirable aerosol of cocaine base. Pharmacol Biochem Behav 1988; 29: 93 - 95

${ }^{7}$ Warner EA. Cocaine abuse. Ann Intern Med 1993; 119: 226 - 235

${ }^{8}$ Hannan DJ, Adler AG. Crack abuse. Do you known enough about it? Postgrad Med 1990; 88: 141 - 147

${ }^{9}$ Leo T. Frankfurt: Crack City. Journal Frankfurt 2001; 16: 26 -31

${ }^{10}$ Brody SL, Slovis CM, Wrenn KD. Cocaine-related medical problems: consecutive series of 233 patients. Am J Med 1990; 88: 325 - 331

11 Tashkin DP, Khalsa ME, Gorelick D et al. Pulmonary status of habitual cocaine smokers. Am Rev Respir Dis 1992; 145: 92-100

12 Taylor RF, Bernard GR. Airway complications from free-basing cocaine. Chest 1989; 95: 476-477

${ }^{13}$ Susskind H, Weber DA, Volkow ND et al. Increased lung permeability following long-term use of free-base cocaine (crack). Chest 1991; 100: 903-909

${ }^{14}$ Murray RJ, Smialek JE, Golle M et al. Pulmonary artery medial hypertrophy in cocaine users without foreign particle microembolization. Chest 1989; 96: 1050-1053

${ }^{15}$ Kline JN, Hirasuna JD. Pulmonary edema after freebase cocaine smoking - not due to an adulterant. Chest 1990; 97: 1009-1010

${ }^{16}$ Hoffman CK, Goodman PC. Pulmonary edema in cocaine smokers. Radiology 1989; 172: $463-465$

${ }^{17}$ Godwin JE, Harley RA, Miller KS et al. Cocaine, pulmonary hemorrhage, and hemoptysis. Ann Intern Med 1989; 110: 843 - 850

\section{Buchbesprechung}

\section{Springer Großwörterbuch Medizin - Medical Dictionary Deutsch-Englisch/English-German}

\section{P. Reuter}

Berlin Heidelberg New York: Springer Verlag, 2001, 14 Abb., XIII. 1531 S. , 2130 g. Geb. $€ 49,95 /$ SFR 80,00

Wie in allen Biowissenschaften hat sich in der theoretischen wie in der klinischen Medizin das („angloamerican“) Englisch durchgesetzt. Seine Kenntnis ist für den wissenschaftlich tätigen wie für den „praktischen“ (d.h. praktizierenden) Arzt heute unverzichtbar.

Das „Springer Großwörterbuch Medizin/Medical Dictionary“ enthält als erstes umfassendes Fachwörterbuch neben den Übersetzungen auch die Definitionen der deutschen/englischen Stichwörter; es bietet so eine bisher nicht verfügbare Qualität an Information.

Der deutsch-englische Lexikonteil basiert auf dem „Springer Wörterbuch Medizin“; er enthält rd. 45000 Stichwörter mit Definitionen, Synonymen und die Übersetzung des Stichworts. Dabei wurde auch die Rechtschreibereform berücksichtigt wie die „Terminologic Anatomica“. Der englisch-deutsche Teil enthält sogar ca. 50000 Stichwörter, Untereinträge und Anwendungsbeispiele mit mehr als 100000 Übersetzungen - mit Aussprache der englischen Termini und Silbentrennung der Hauptwörter.

Umrechnungstabellen für Maße, Gewichte, Temperaturen sowie eine Tabelle mit Normalwerten klinisch wichtiger Parameter ergänzen - wie auch 14 anatomische Abbildungen - den „Übersetzer-Komplex“.

Im Teil „Abkürzungen und Akronyme/Abbreviations and Acronyms" werden mehr als 5000 Abkürzungen, Symbole und Zeichen aufgeführt. Bei der heute frustrierenden Vermehrung von „Akronymen“ ist natürlich eine Perfektion unmöglich: so werden z.B. für „H." acht englische und 10 deutsche Definitionen angegeben; bei „CRS“ nur das „Chinese Restaurant Syndrome“, nicht aber die „Camp Reception Station“ (Krankensammelstelle), „Colon and Rectal Surgery“, „Congenital Rubella Syndrome“, „Cottbus Reinfarction Study“ und „Croix Rouge Suisse“ erwähnt und das „ARDS“ wie „COLD/COPD“ (und andere) fehlen überhaupt.

Insgesamt ein wertvolles, wichtiges, ja unverzichtbares Nachschlagewerk! Zur Nutzung des 2,13 kg schweren Buches ist ein Stehpult vorteilhaft.

Heinz S. Fuchs, Bad Godesberg 